Supporting Information

\title{
Thermally Sensitive Molecularly Imprinted Polymers on Mesoporous Silica Nanospheres: Preparation, Characterization and Properties as Novel Adsorbents for Dichlorophen
}

Jifeng Guo, ${ }^{\text {a, }}{ }^{*}$ Hui Chen, ${ }^{\text {a, b }}$ Xiao Wei, ${ }^{\text {a, b* }}$

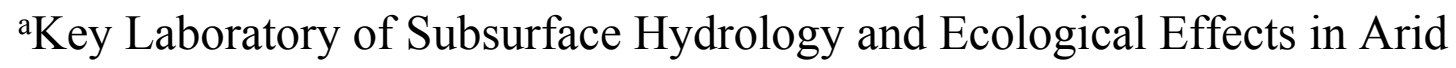
Region, Ministry of Education, Chang'an University, Xi'an 710054,

China

${ }^{\mathrm{b} S c h o o l ~ o f ~ E n v i r o n m e n t a l ~ S c i e n c e ~ a n d ~ E n g i n e e r i n g, ~ C h a n g ' a n ~}$ University, Xi'an 710054, China

*Corresponding author: Tel: +86 029 82339956; Fax: +86 029 82339281;

E-mail address: guojifeng@chd.edu.cn (J.F. Guo).chdwx@chd.edu.cn (X. Wei). 

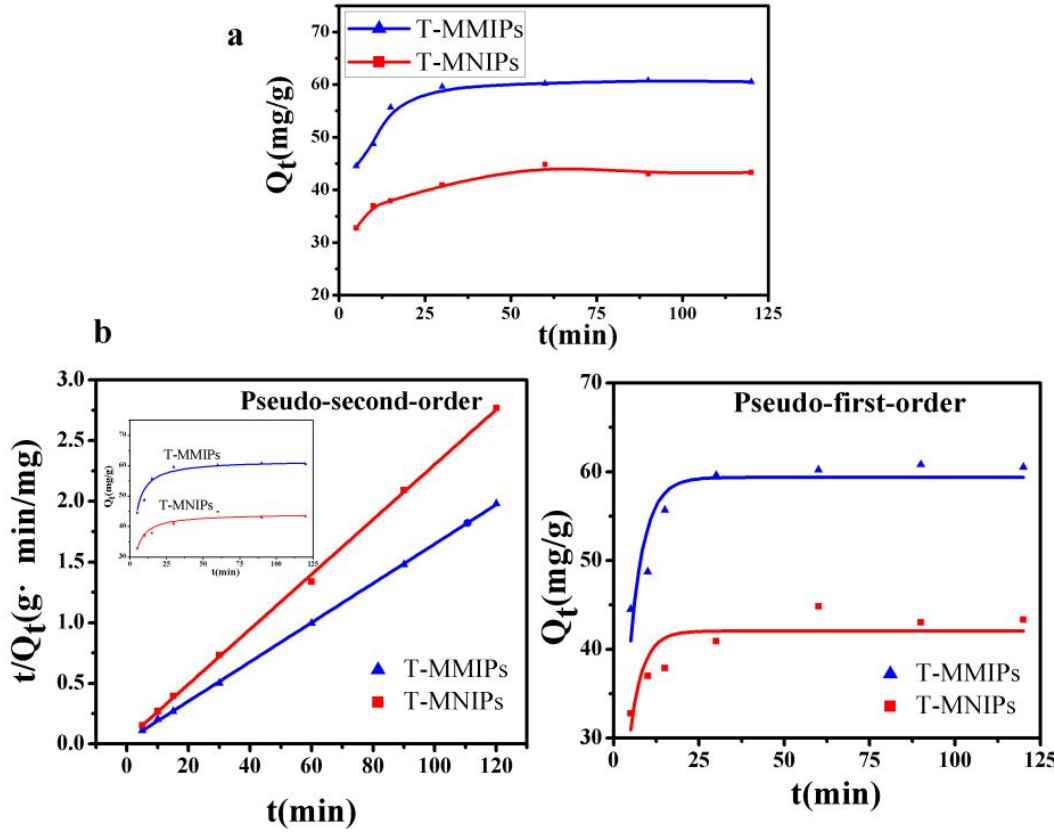

Figure S1. (a) Dynamic adsorption curves, (b) kinetic fitting of T-MMIPs and T-MNIPs

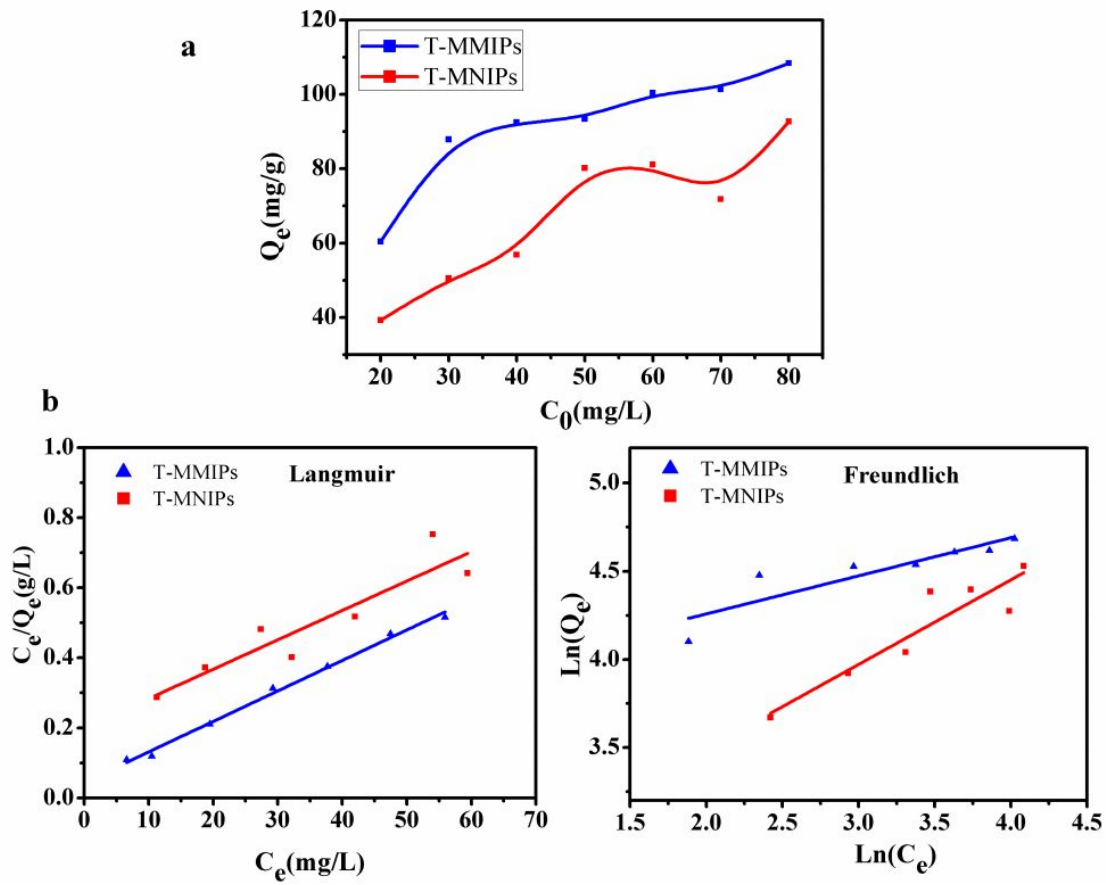

Figure S2. (a) Static binding isotherms and (b) isotherms linear fitting of T-MMIPs and T-MNIPs 

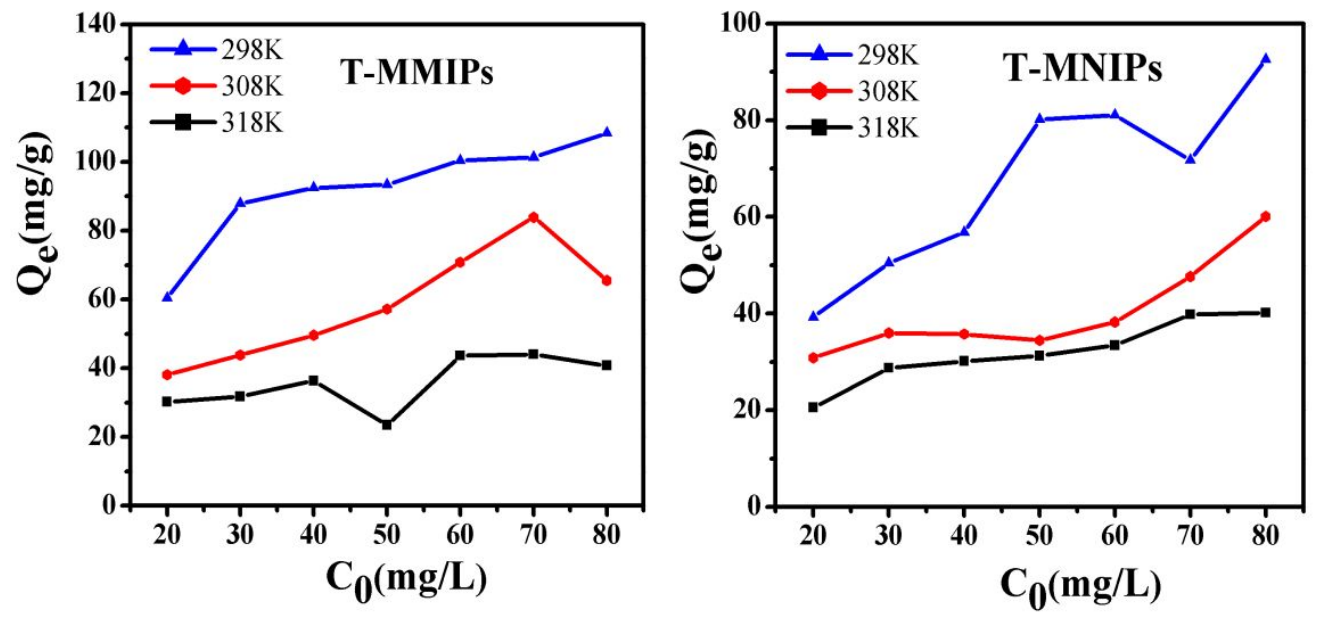

Figure S3. Temperature sensibility of T-MMIPs and T-MNIPs 
Table S1. List and information of chemicals

\begin{tabular}{|c|c|c|c|c|}
\hline component & molecular formula & CAS Reg. No. & suppliers & $\begin{array}{l}\text { mass } \\
\text { fraction }\end{array}$ \\
\hline Cetrimonium Bromide & $\mathrm{C}_{19} \mathrm{H}_{42} \mathrm{BrN}$ & $57-09-0$ & $\begin{array}{c}\text { Tianjin Damao Chemical } \\
\text { Reagent Factory }\end{array}$ & $\geqslant 99 \%$ \\
\hline $\begin{array}{l}\text { 3-(Methacryloyloxy) } \\
\text { propyltrimethoxysilane }\end{array}$ & $\mathrm{C}_{10} \mathrm{H}_{20} \mathrm{O}_{5} \mathrm{Si}$ & $2530-85-0$ & $\begin{array}{c}\text { Shandong Yousuo Chemical } \\
\text { Technology Co., Ltd. }\end{array}$ & $>97 \%$ \\
\hline $\mathrm{N}$-isopropylacrylamide & $\mathrm{C}_{6} \mathrm{H}_{11} \mathrm{NO}$ & $2210-25-5$ & $\begin{array}{c}\text { Shanghai Macklin } \\
\text { Biochemical Co., Ltd. }\end{array}$ & $\geqslant 98 \%$ \\
\hline $\begin{array}{l}\text { Ethylene glycol } \\
\text { dimethacrylate }\end{array}$ & $\mathrm{C}_{4} \mathrm{H}_{6} \mathrm{O}_{2}$ & $79-41-4$ & Aladdin Reagent CO. Ltd. & $>99 \%$ \\
\hline $\begin{array}{c}2,2^{\prime} \text {-Azobis } \\
\text { (2-methyl-propionitrile) }\end{array}$ & $\mathrm{C}_{8} \mathrm{H}_{12} \mathrm{~N}_{4}$ & $78-67-1$ & $\begin{array}{c}\text { Chengdu Kelong Chemical } \\
\text { Reagent Factory }\end{array}$ & $\geqslant 98 \%$ \\
\hline Acrylamide & $\mathrm{C}_{3} \mathrm{H}_{5} \mathrm{NO}$ & $79-06-1$ & Aladdin Reagent CO. Ltd. & $\geqslant 99 \%$ \\
\hline Dichlorophen & $\mathrm{C}_{13} \mathrm{H}_{10} \mathrm{Cl}_{2} \mathrm{O}_{2}$ & $97-23-4$ & Aladdin Reagent CO. Ltd. & $>98 \%$ \\
\hline 2,4-dichlorophenol & $\mathrm{C}_{6} \mathrm{H}_{4} \mathrm{Cl}_{2} \mathrm{O}$ & $120-83-2$ & Aladdin Reagent CO. Ltd. & $>98 \%$ \\
\hline 2,6-dichlorophenol & $\mathrm{C}_{6} \mathrm{H}_{4} \mathrm{Cl}_{2} \mathrm{O}$ & $87-65-0$ & Aladdin Reagent CO. Ltd. & $>99 \%$ \\
\hline Sulfonamide & $\mathrm{C}_{6} \mathrm{H}_{8} \mathrm{~N}_{2} \mathrm{O}_{2} \mathrm{~S}$ & $63-74-1$ & $\begin{array}{l}\text { Tianjin Komiou Chemical } \\
\text { Reagent Factory }\end{array}$ & $>98 \%$ \\
\hline
\end{tabular}

Table S2. Parameters of kinetic models for DCP adsorption onto T-MMIPs and T-MNIPs

\begin{tabular}{|c|c|c|c|c|c|c|c|c|}
\hline \multirow{2}{*}{ Samples } & \multirow{2}{*}{$\mathrm{T}(\mathrm{K})$} & \multirow{2}{*}{$\begin{array}{l}Q_{e, \exp } \\
(\mathrm{mg} / \mathrm{g})\end{array}$} & \multicolumn{3}{|c|}{ Pseudo-first-order } & \multicolumn{3}{|c|}{ Pseudo-second-order } \\
\hline & & & $\begin{array}{c}\mathrm{Q}_{\mathrm{e}, \mathrm{c}} \\
(\mathrm{mg} / \mathrm{g})\end{array}$ & $\begin{array}{c}\mathrm{k}_{1} \\
(\mathrm{~L} / \mathrm{min}) \\
\end{array}$ & $\mathrm{R}^{2}$ & $\begin{array}{c}\mathrm{Q}_{\mathrm{e}, \mathrm{c}} \\
(\mathrm{mg} / \mathrm{g})\end{array}$ & $\begin{array}{c}\mathrm{k}_{2} \\
(\mathrm{~g} \cdot \mathrm{min} / \mathrm{mg})\end{array}$ & $\mathrm{R}^{2}$ \\
\hline T-MMIPs & 298 & 60.82 & 59.37 & 0.23 & 0.9044 & 45.60 & 0.21 & 0.7646 \\
\hline T-MNIPs & 298 & 43.54 & 42.05 & 0.26 & 0.8037 & 44.19 & 0.01 & 0.9991 \\
\hline
\end{tabular}

Table S3. Parameters of isotherm constants for DCP adsorption onto T-MMIPs and T-MNIPs

\begin{tabular}{|c|c|c|c|c|c|c|c|c|}
\hline \multirow{2}{*}{ Samples } & \multirow{2}{*}{$\mathrm{T}(\mathrm{K})$} & \multirow{2}{*}{$\begin{array}{c}\mathrm{Q}_{\mathrm{e}} \\
(\mathrm{mg} / \mathrm{g})\end{array}$} & \multicolumn{3}{|c|}{ Langmuir } & \multicolumn{3}{|c|}{ Freundlich } \\
\hline & & & $\begin{array}{c}\mathrm{Q}_{\mathrm{m}} \\
(\mathrm{mg} / \mathrm{g})\end{array}$ & $\begin{array}{c}\mathrm{K}_{\mathrm{L}} \\
(\mathrm{L} / \mathrm{mg})\end{array}$ & $\mathrm{R}^{2}$ & $\begin{array}{c}\mathrm{K}_{\mathrm{F}} \\
{[(\mathrm{mg} / \mathrm{g})(\mathrm{L} / \mathrm{mg})]}\end{array}$ & $1 / \mathrm{n}$ & $\mathrm{R}^{2}$ \\
\hline T-MMIPs & 298 & 108.35 & 114.94 & 0.19 & 0.9933 & 45.604 & 0.21 & 0.7646 \\
\hline T-MNIPs & 298 & 92.66 & 94.876 & 0.05 & 0.8306 & 12.591 & 0.47 & 0.8343 \\
\hline
\end{tabular}


Table S4. Adsorption selectivity of T-MMIPs and T-MNIPs

\begin{tabular}{|c|c|c|c|c|c|c|c|}
\hline \multirow{2}{*}{ Antibiotics } & \multicolumn{3}{|c|}{ T-MMIPs } & \multicolumn{3}{|c|}{ T-MNIPs } & \multirow{2}{*}{$\mathrm{k}^{\prime}$} \\
\hline & $\begin{array}{c}\mathrm{C}_{\mathrm{e}} \\
(\mathrm{mg} / \mathrm{L})\end{array}$ & $\begin{array}{c}\mathrm{K}_{\mathrm{d}} \\
(\mathrm{L} / \mathrm{g})\end{array}$ & & $\begin{array}{c}\mathrm{C}_{\mathrm{e}} \\
(\mathrm{mg} / \mathrm{L})\end{array}$ & $\begin{array}{c}\mathrm{K}_{\mathrm{d}} \\
(\mathrm{L} / \mathrm{g})\end{array}$ & $\mathrm{k}$ & \\
\hline DCP & 29.24 & 3.19 & - & 32.19 & 2.49 & - & - \\
\hline 2,4-DCP & 44.67 & 0.53 & 5.95 & 46.34 & 0.35 & 7.01 & 0.84 \\
\hline 2,6-DCP & 46.73 & 0.31 & 10.17 & 47.71 & 0.21 & 11.58 & 0.87 \\
\hline SN & 48.54 & 0.13 & 23.66 & 49.43 & 0.05 & 47.05 & 0.50 \\
\hline
\end{tabular}

